\title{
Optimization of MUSIC and Improved MUSIC Algorithm to Estimate Direction of Arrival
}

\author{
Pooja Gupta \\ School of Electronics, KIIT University, Patia 751024, Bhubaneswar, Odisha, India \\ Email: pooja.guptafet@kiit.ac.in \\ Vijay Verma \\ School of Electronics, KIIT University, Patia 751024, Bhubaneswar, Odisha, India \\ Email:vijay.vermafet@kiit.ac.in
}

\begin{abstract}
The signal processing applications are limited with high-resolution signal parameter estimation. Therefore the Direction of Arrival estimation algorithm needs to be effective and efficient in order to improve the performance of smart antennas. This paper presents the simulation for a subspace based DOA estimation algorithm with high resolution. MUSIC (Multiple signal classification) and the IMUSIC (Improved MUSIC) are presented and optimized by varying various parameters. The basic MUSIC algorithm is ineffective in estimating the incoming coherent signals. The new improved MUSIC algorithm overcomes this ineffectiveness and correctly estimates the related signals with improved accuracy. The improved version of MUSIC algorithm is brought about by taking into account the conjugate of the data matrix for MUSIC algorithm and then reconstructing it. The various factors like the number of array elements, number of snapshots, varying the distance between array elements, varying SNR and the difference in arrival angles can bring about better resolutions. The comparisons for MUSIC and Improved MUSIC algorithm are widely discussed.
\end{abstract}

Index Terms-DOA, MUSIC, ML, IMUSIC, ULA, ESPRIT.

\section{INTRODUCTION}

The smart antenna system detects the emitted narrow band signals from different sources using the sensor arrays by applying specific estimation algorithms. The array signal processing works on processing and strengthening the useful signals that are received by the antenna elements, then taking measures for diminishing noise and interference, and simultaneously collecting needful signal parameters in order to carry out the estimation process .

The various engineering applications like the radio, sonar, wireless communication, astronomy, earthquake, medicines, tracking and other emergencies have a vital importance for the Direction of Arrival (DOA) estimation algorithms [1]. Smart antennas provide higher communication capacity by suppressing multipath signals and interference. The resolution of the estimation process can be enhanced by using an array of antenna system rather than a single antenna [2] as the system provides spatial sampling. The Barlett, MUSIC, MVDR, Estimation of signal Parameter through Rotational Invariance Technique (ESPRIT), Capon, Maximum Likelihood (ML) techniques [3] and Min-norm are the various resolution algorithms.

The spatial spectrum comprises the target stage, observation stage and the estimation stage [4] and assume that the signals are distributed in the entire space in all the directions. This signal spatial spectrum is exploited to obtain the Direction of Arrival of the incoming source signals. ESPRIT [5] and the MUSIC (Multiple Signal Classification) [6-7] are the two most widely used subspaces based spectral estimation techniques which work on the Eigen value decomposition technique. The covariance matrices of the signals form the base for the subspace based approaches. As the ESPRIT estimation algorithm is applicable only to the array structures with peculiar geometries [8], the MUSIC algorithm is found to be the most classic and accepted parameter estimation technique that can be used for both uniform and nonuniform linear arrays. It works with the Uniform Linear Array (ULA) where the Nyquist criterion forms the basis for the placement of the array elements. The nonuniform array designing [9] is quite tedious.

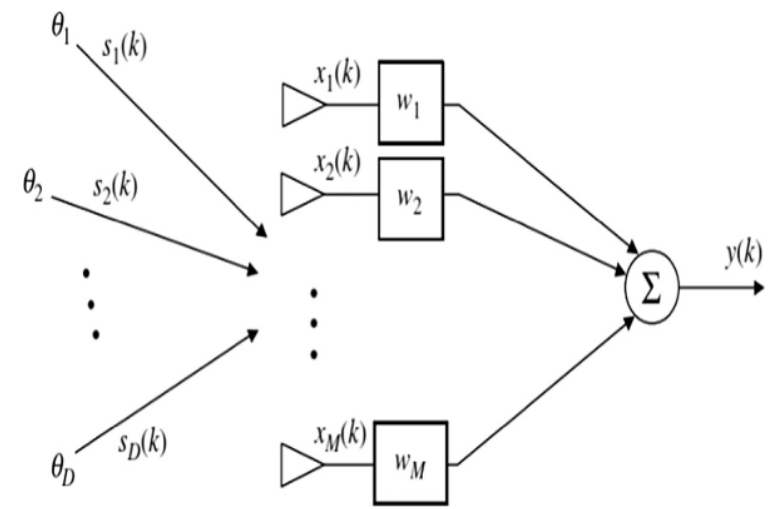

Fig.1. The array antenna model for DOA Estimation Algorithms.

A simple arrangement of the antenna array receive model is shown in the above figure. The estimation algorithms are supposed to compute the number of 
incident signals on the sensor array, their corresponding strengths and the direction of arrival i.e. the incidence angle of the incoming signal. For carrying out the estimation process we first need to uniformly sample the bearing space to fetch many of the discrete angles, and then we need to follow the assumption that the source and noise signals are arriving from every small bearing angles and the estimation algorithm computes the angle of signal corresponding to stronger power.

The simulations on varying the factors are carried out using MATLAB which specify that the efficiency and resolution of the obtained spectrum using MUSIC algorithm can be increased by varying various parameters like the spacing between the array elements, number of array elements, number of snapshots and the signal incidence angle difference. The estimation of the related signals is not that efficient by using the conventional MUSIC algorithm, and this algorithm is found to have a declining performance when the antenna array has phase and amplitude errors. An improved MUSIC algorithm which is brought about by taking into account the conjugate of the data matrix for MUSIC algorithm and then reconstructing it is also broadly discussed. This improved version of the MUSIC algorithm solves the problem for the incoming signals that are coherent. This algorithm is found to solve all the such estimation errors where the conventional MUSIC lags. This paper widely discusses the performance of the above two mentioned estimation algorithms on varying the factors related to the estimation scenario.

The paper is organized as follows. Section II describes the mathematics behind the array sensor system. Section III gives the MUSIC algorithm and in IV the parameters influencing the performance of MUSIC algorithm are discussed. Section $\mathrm{V}$ focuses on the detection of coherent signals and then the following section describes the Improved MUSIC algorithm. Finally section VI concludes this article by drawing inferences on study.

We are considering $F$ narrow banded source signals with the same centre frequency $f_{0}$ which are impinging on an array with D number of sensor elements. The number $\mathrm{D}$ is always kept greater than the number of incident source signals and the array elements are linearly spaced with equal distances between consecutive elements. Restricting the number of sensors to be greater than the number of signals being incident i.e. (D>F) for a better estimation result we suppose that the $M$ incoming signals will be incident on the sensor array with azimuth angles $\theta_{\mathrm{k}}$, where $\mathrm{k}$ varies from 1 to $\mathrm{F}$. The consecutive elements of the array are placed such that they maintain a distance of half of the received signal wavelength [10]. The space matrix $\left[d_{1} d_{2} \ldots . d_{D-1}\right]$ represents the consecutive elements of the antenna array.

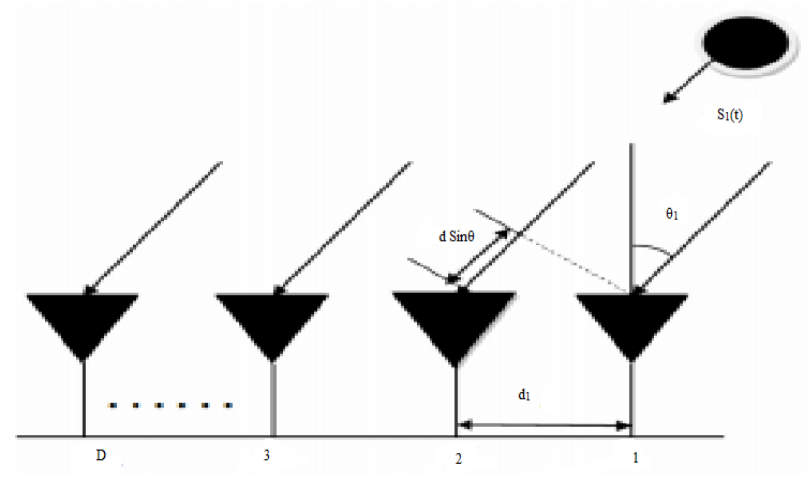

Fig.2. The antenna system model for DOA Estimation

The figure shown gives the structure of an uniform antenna array i.e. $d_{1}=d_{2}=d_{D-1}$ which lies in the same plane as the incoming signals.

If the source signal has a phase of $\phi(t)$ and amplitude to be $a_{a m p}(t)$, The complex form representation of the source signal is :

$$
S_{s}(t)=a_{a m p}(t) e^{j\left(f_{0}(t)+\phi(t)\right)}
$$

If the first element of the antenna array is taken as the reference element, the signal sensed because of the $\mathrm{p}^{\text {th }}$ signal source [11] by the $\mathrm{k}^{\text {th }}$ array element will be represented as:

$$
\mathrm{S}_{\mathrm{p}} \mathrm{e}^{\frac{-\mathrm{j} 2 \pi \mathrm{d}_{\mathrm{k}}(\mathrm{k}-1) \sin \theta_{\mathrm{p}}}{\lambda}} \text { where } 1 \leq \mathrm{p} \leq \mathrm{F}
$$

The overall sensing for all the incoming signals done at the $\mathrm{k}^{\text {th }}$ array (where $1 \leq \mathrm{k} \leq \mathrm{D}$ ) is calculated as :

$$
\mathrm{O}_{\mathrm{p}}(\mathrm{t})=\sum_{\mathrm{p}=1}^{\mathrm{F}} \mathrm{e}^{\frac{-\mathrm{j} 2 \pi \mathrm{d}_{\mathrm{k}}(\mathrm{k}-1) \sin \theta_{\mathrm{p}}}{\lambda}} \mathrm{s}_{\mathrm{p}}(\mathrm{t})+\mathrm{n}_{\mathrm{k}}(\mathrm{t})
$$

Taking the separation between the $(\mathrm{k}-1)^{\mathrm{th}}$ and the $\mathrm{k}^{\text {th }}$ array elements to be $d_{k}$, and $S_{p}(t)$ representing the $p^{\text {th }}$ signal that is being incident on the antenna array, and $n_{k}$ representing the noise signal that is being sensed by the $\mathrm{k}^{\text {th }}$ element of antenna array. We denote a matrix $\mathrm{O}(\mathrm{t})$ representing the received signal that comprises of all the information obtained by the overall D array elements. The matrix representation is given as:

$$
\mathrm{O}(\mathrm{t})=\mathrm{AS}_{\mathrm{s}}(\mathrm{t})+\mathrm{N}(\mathrm{t})
$$

' $A$ ' in the above equation represents a steering vector matrix and $\mathrm{N}(\mathrm{t})$ gives the total noise that is received by all the array elements. The received signal by the array elements is:

$$
\mathrm{O}(\mathrm{t})=\left[\mathrm{O}_{1}(\mathrm{t}) \mathrm{O}_{2}(\mathrm{t}) \ldots \mathrm{O}_{\mathrm{D}}(\mathrm{t})\right]^{\mathrm{T}}
$$


Now calculating the matrix representation for the ' $F$ ' signal sources that are being incident on sensor array and the steering vector matrix ' $A$ ' $[11]$ :

$$
\begin{aligned}
& S_{s}(t)=\left[\begin{array}{lll}
S_{1}(t) & S_{2}(t) \ldots S_{F}(t)
\end{array}\right]^{T} \\
& \mathrm{~A}=\left[\beta\left(\theta_{1}\right) \beta\left(\theta_{2}\right), \ldots \beta\left(\theta_{\mathrm{F}}\right)\right]
\end{aligned}
$$

The above two matrices given in equations (6) and (7) form the signal subspace. And in the following equation $\beta\left(\theta_{\mathrm{p}}\right)$ gives the number of source signals that are being incident as $\beta\left(\theta_{\mathrm{p}}\right)=$

$$
\left[1 \mathrm{e}^{-\mathrm{j} \frac{2 \pi \mathrm{d}_{1}}{\lambda} \sin (\theta \mathrm{p})} \ldots \mathrm{e}^{-\mathrm{j}(\mathrm{D}-1) \frac{2 \pi \mathrm{d}_{\mathrm{D}-1}}{\lambda} \sin (\theta \mathrm{p})}\right]^{\mathrm{T}}
$$
F.

The value of $\mathrm{p}$ in the above equation ranges from 1 to

\section{THE MUSIC ALGORITHM}

Estimation approach known as Multiple Signal Classification (MUSIC) algorithm in 1979[12].This algorithm is similar in character with the maximum likelihood method and is basically an one-dimensional representation of the maximum entropy. The basic approach of this algorithm is to separate the signal from the noise using the Eigen value decomposition of the received signal covariance matrix. It uses the orthogonality property of both the signal and noise space. Then the estimation is brought about by using one of these subspaces and considering that the noise in each channel is highly uncorrelated. As this algorithm takes uncorrelated noise into account, the generated covariance matrix is diagonal in nature. Here the signal and the noise subspaces are computed using the matrix algebra and are found to be orthogonal to each other. Therefore this algorithm exploits the orthogonality property to isolate the signal and the noise subspaces.

There are a variety of algorithms for carrying out the estimation process, out of which this paper focuses on the most accepted and widely used Multiple Signal Classification algorithm. It decomposes the obtained data correlation matrix into two orthogonal subspaces, namely the signal and the noise subspaces. And then it uses of these subspaces to estimate the direction of the incoming signal. The obtained data correlation matrix actually forms the base for the MUSIC algorithm. We then need to search through the entire steering vector matrix and then try bringing out those steering signals and noise vectors which are exactly orthogonal. Let ' $\mathrm{C}_{\mathrm{J}}$ ' be the covariance matrix for the received data $\mathrm{O}$, where covariance matrix is the expectance of the matrix with its Hermitian equivalent.

$$
\mathrm{C}_{\mathrm{O}}=\mathrm{E}\left[\mathrm{OO}^{\mathrm{H}}\right]
$$

Now using equation (5) for the value of $\mathrm{O}$ we get:

$$
\mathrm{C}_{\mathrm{O}}=\mathrm{E}[(\mathrm{AS}+\mathrm{N})(\mathrm{AS}+\mathrm{N})]
$$

$$
\begin{gathered}
=\mathrm{AE}\left[\mathrm{SS}^{\mathrm{H}}\right] \mathrm{A}^{\mathrm{H}}+\mathrm{E}\left[\mathrm{NN}^{\mathrm{H}}\right] \\
=\mathrm{AC}_{\mathrm{O}} \mathrm{A}^{\mathrm{H}}+\mathrm{C}_{\mathrm{N}}
\end{gathered}
$$

$\mathrm{C}_{\mathrm{N}}$ is the correlation matrix for noise and it can be expressed as:

$$
\mathrm{C}_{\mathrm{N}}=\sigma^{2} \mathrm{I}
$$

I in the above equation represents an unit matrix for the antenna array $\mathrm{D} * \mathrm{D}$. As the signals practically are associated with some noise, we need to compute the correlation matrix taking noise into account, so the new modified matrix is:

$$
\mathrm{C}_{\mathrm{O}}=\mathrm{AC}_{\mathrm{S}} \mathrm{A}^{\mathrm{H}}+\mathrm{C}_{\mathrm{N}}
$$

$\mathrm{C}_{\mathrm{S}}$ here denotes the source correlation matrix, $\mathrm{C}_{\mathrm{N}}$ denotes the noise correlation matrix and A denotes the steering vector matrix. In order to distinguish the signal sources from the noise sources we have to carry out the eigen value decomposition for the calculated covariance matrix $C_{S}$ which will result in ' $D$ ' eigen values where ' $F$ ' number of larger eigen values corresponds the signal sources and the noise sources are given by the remaining smaller D-F eigen values. Let $\mathrm{B}_{\mathrm{S}}$ and $\mathrm{B}_{\mathrm{N}}$ be the basis for the signal and the noise subspaces respectively. Now the decomposed form of the correlation matrix can be given as:

$$
\begin{gathered}
\mathrm{C}_{\mathrm{O}}=\mathrm{B}_{\mathrm{S}} \sum \mathrm{B}_{\mathrm{S}}{ }^{\mathrm{H}}+\mathrm{B}_{\mathrm{N}} \sum \mathrm{B}_{\mathrm{N}}{ }^{\mathrm{H}} \\
\beta^{\mathrm{H}}(\theta) \mathrm{B}_{\mathrm{N}}=0
\end{gathered}
$$

Equation (14) holds true as the MUSIC estimation approach is based on the orthogonality of the signal and noise subspaces. Now the incident signal sources and the noise subspaces can be arranged so as to obtain the angle for the direction of the arriving signal as shown in the following equation:

$$
\theta_{\text {MUSIC }}=\operatorname{argmin} \cdot \beta^{\mathrm{H}}(\theta) \mathrm{B}_{\mathrm{N}} \mathrm{B}_{\mathrm{N}}{ }^{\mathrm{H}} \beta(\theta)
$$

As finding peaks in the spectral estimation plot can be the best way to identify the angles from which the signals are arriving, we need to work on the reciprocal value of $\theta_{\text {MUSIC }}$ in order to obtain the maximum spectrum values. There are several approaches namely the linear prediction, eigen analysis, beam forming, array correlation matrix, maximum likelihood, minimum variance, MUSIC etc for defining the pseudo spectrum function ( $\left.\mathrm{P}_{\text {MUSIC }}\right)$. Now the reciprocal representation of the above equation to obtain peaks in a spectral estimation plot is given:

$$
\mathrm{P}_{\text {MUSIC }}=\frac{1}{\beta^{\mathrm{H}}(\theta) \mathrm{B}_{\mathrm{N}} \mathrm{B}_{\mathrm{N}}{ }^{\mathrm{H}} \beta(\theta)}
$$

This above mentioned will give sharp and high peaks when $\theta$ is exactly equal to that of the direction of arrival of the signal source. The $\mathrm{F}$ higher peaks are of greater power [13] and corresponds to the estimated arrival angle. 
The entire algorithm can be summarized using a flow chart as shown below:

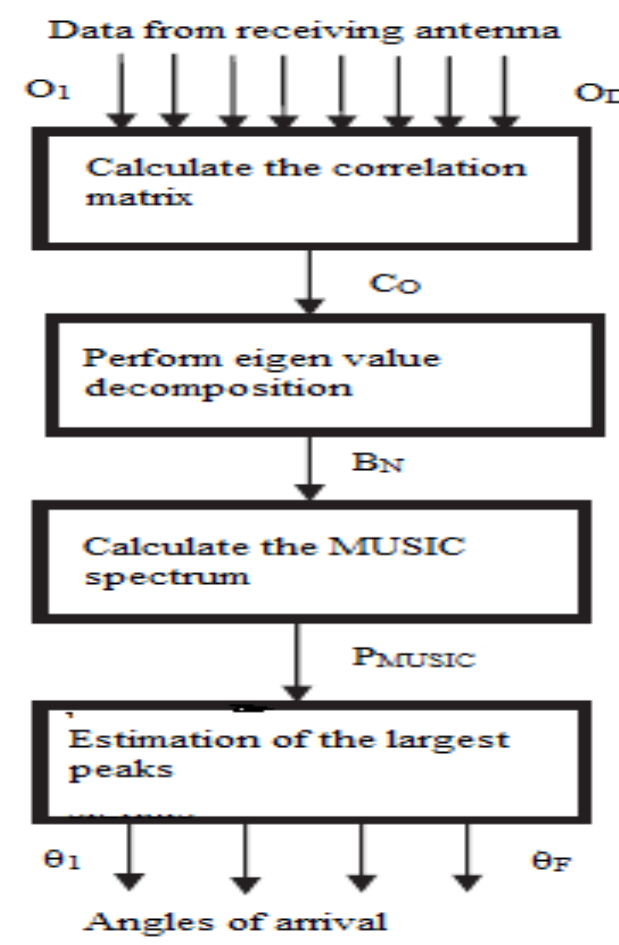

Fig.3. Flow chart summarizing MUSIC algorithm.

\section{Simulation Result FOR A BASIC MUSIC ALGORITHM}

Fill For carrying out the basic standard simulation for MUSIC algorithm the signals considered are narrow banded and non-coherent, the distance between the antenna arrays is taken to be half the wavelength of the received signal .The standard number of array elements ' $\mathrm{D}$ ' used is 10 , SNR considered is $10 \mathrm{~dB}$ and the number of snapshot taken is 200 . The noise here is additive white Gaussian noise. We have considered the two incoming signals with arrival angles $30^{\circ}$ and $70^{\circ}$. The following graph shows the MATLAB simulation for the MUSIC algorithm considering the above mentioned specifications:

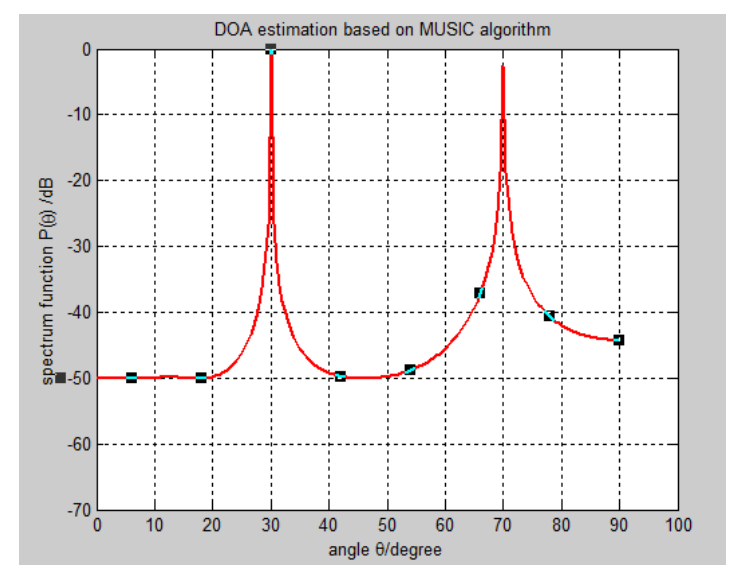

Fig.4. Spatial spectrum for MUSIC algorithm
The independent spectrum peaks in the graph corresponds to $30^{\circ}$ and $70^{\circ}$ stating that the MUSIC algorithm is efficient enough to estimate the arrival angles of the signal sources.

\section{FACTORS INFLUENCING THE PERFORMANCE OF MUSIC ALGORITHM}

There are various parameters that can influence the performance of MUSIC algorithm. The following sections describe those factors that can bring about better resolution in the MUSIC algorithm

\section{A. Dependence of SNR on estimation process}

We carry out the simulation by keeping all the specifications to be the same as used for the basic MUSIC and varying SNR successively as 0, 10, 15 and $20 \mathrm{~dB}$

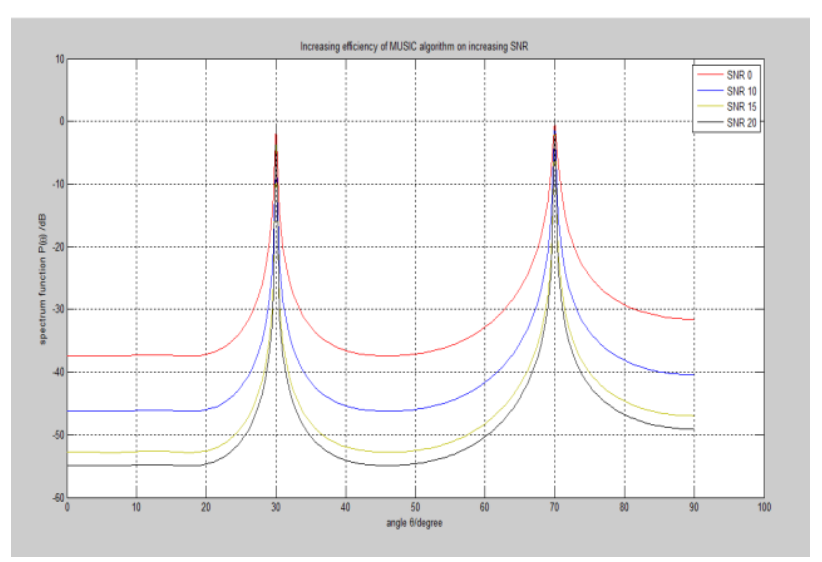

Fig.5. Efficiency result on varying the SNR

The color red, blue, green and black corresponds to the estimation results for $0,10,15$ and $20 \mathrm{~dB}$ SNR. The peaks are found to be more and sharper and distinguished as the value of SNR gets higher. The estimation plots covering a larger range of the spectral function have better efficiency and resolution. Hence improving the performance of the estimation process in the low SNR condition is currently the main research topic.

\section{B. Dependence of the number of sensor elements on the estimation process}

On keeping the same specifications and varying the number of sensor elements as 10,20,50 and 100, we find that the peaks corresponding to the estimation with higher number of array elements are more and more sharp and prominent. We now carry out a simulation with varying number of sensor elements. The estimation graph for the same is shown: 


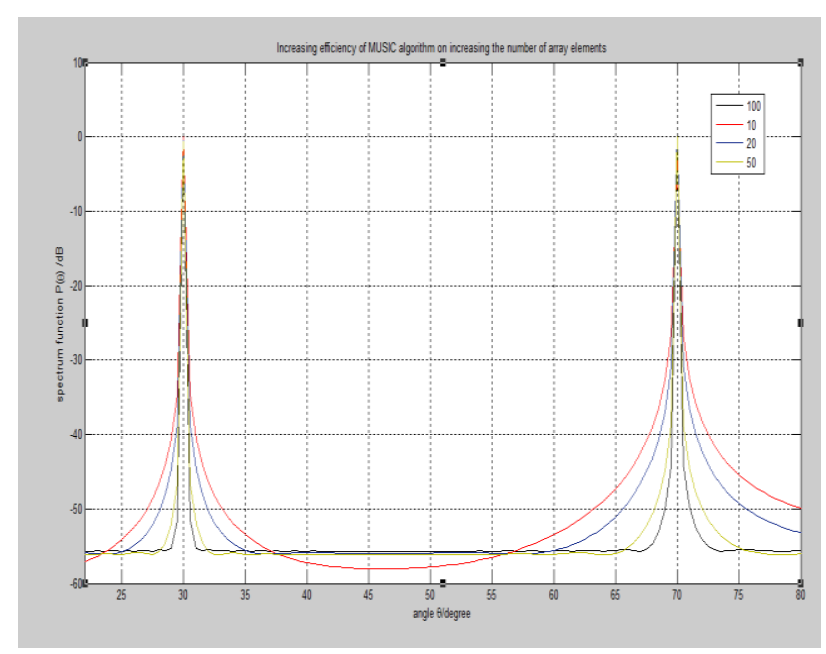

Fig.6. Efficiency results on varying the number of sensor elements.

The red, blue and green curve represents the simulations for 10, 20 and 50 number of array elements respectively. The black color showing the best sharpest curves corresponds to a simulation with 100 array elements. Hence with an increase in the number of sensing elements the estimation process goes more and more efficient. But increasing the sensing elements recklessly is not economical.

\section{Dependence of interelement spacing on estimation process}

The MUSIC algorithm is efficient when the array model is an uniform linear array where the inter element spacing is the same between all the consecutive sensors. The standard simulation considers that the elements are equidistantly placed with a gap of lambda/2, where lambda is the wavelength of the signal. We then carry out the simulation by keeping the uniforms inter element distance to vary as lambda/6 and lambda/4 and lambda/2. The red, blue and black curves corresponds to simulations with inter element spacing as lambda/6, lambda/4 and lambda/2. It is clearly seen that an increase in the spacing within a maximum range of lambda/2 provide more prominent and sharper peaks.

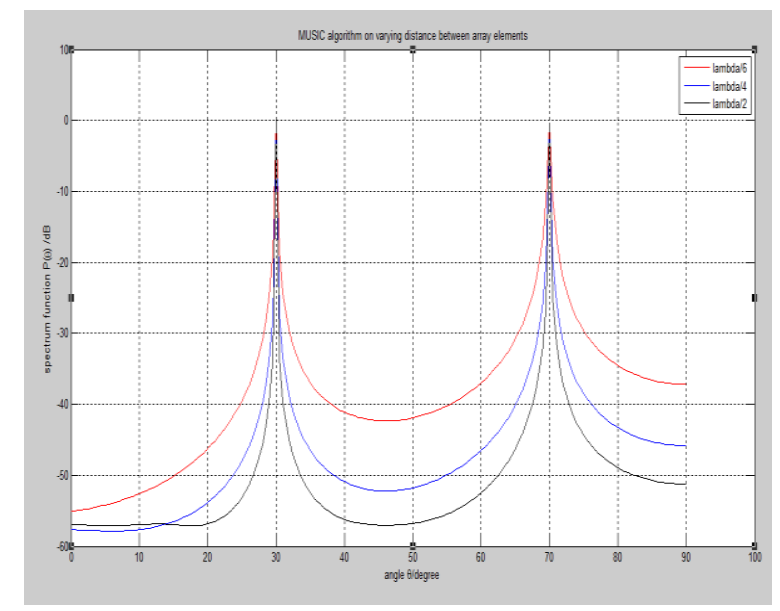

Fig.7. Enhancing efficiency by increasing the inter element spacing within lambda/2.
The red, blue and black curves corresponds to simulations with inter element spacing as lambda/6, lambda/4 and lambda/2. It is clearly seen that an increase in the spacing within a maximum range of lambda/2 provide more prominent and sharper peaks.

Now when the inter element spacing is increased beyond lambda/2, we observe the following:

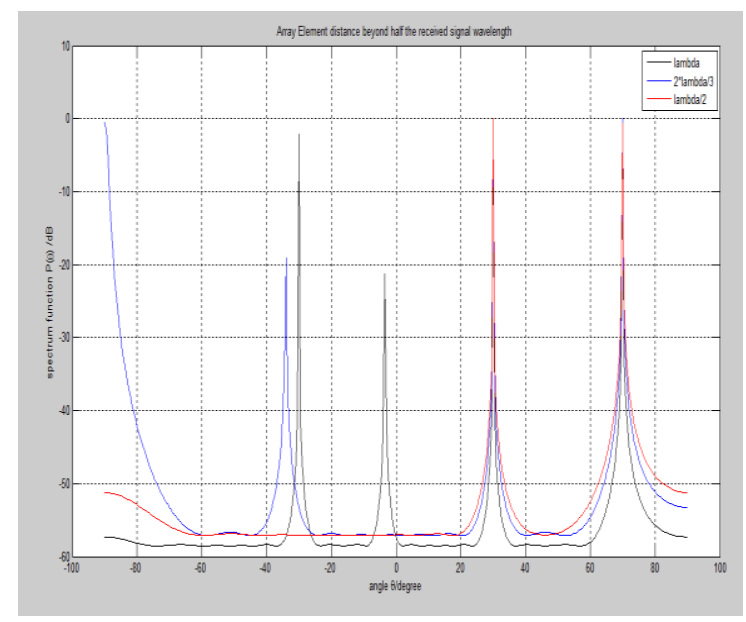

Fig.8. False peaks on increasing the inter element spacing beyond lambda/2.

The estimation algorithm 'MUSIC' produces false peaks when the inter element distance is increased beyond lambda/2. The blue and black curves correspond to an element spacing of $2 * l a m b d a / 3$ and lambda. Hence the best optimized results for the MSUIC algorithm is obtained with an inter element spacing of lambda/2.

D. Dependence of estimation process on the number of snapshots.

The number of snapshots for retrieving the data to find estimation results on varying the number of snapshots is shown below. Te number of snapshot is varied as 10, 100 and 200. The red, blue and black curve corresponds to 10 , 100 and 200 number of snapshots respectively. With other conditions remaining unchanged we find that the beam width of the direction of arrival estimation spectrum becomes narrower and the accuracy is enhanced.

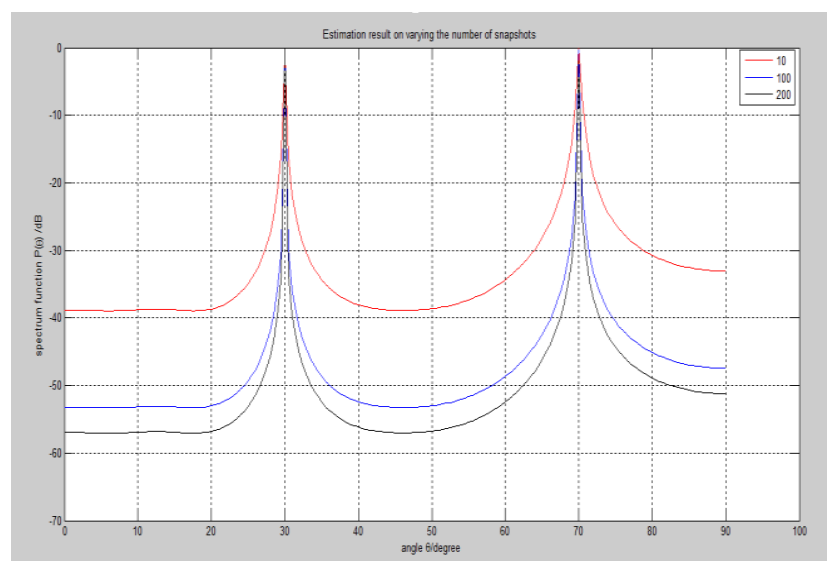

Fig.9. Enhancing efficiency by the number of snapshots. 
The curves corresponding to higher number of snapshots cover larger spectrum ranges. The number of snapshots can be expanded in order to enhance the accuracy, but with increase in snapshots the number of calculations to be carried out also increases. So we need to work with a reasonable number of snapshots that ensures accuracy along with which it minimizes the computation.

\section{DETECTION OF COHERENT Signals}

Estimation algorithms because of its sharp needle spectrum which can estimate the independent source signals precisely. It provides unbiased estimation results in many practical applications, and is even found to perform well in a multiple signal environment. This algorithm achieves high resolution in DOA [14] only when the incoming signals are non-coherent. The results and simulations discussed in the above sections consider that that the incoming signals that are being incident are not related and hence are not generated from the same source. The performance of MSUIC algorithm for the incoming non-coherent signals is quite satisfactory. Now we carry out simulations corresponding to the signals which are coherent and thus are related. Considering the number of array elements to be 10 , the incoming signals to be arriving with angles $30^{\circ}$ and $70^{\circ}$, SNR to be $10 \mathrm{~dB}$, the inter element spacing to be half the signal wavelength, the number of snapshots to be 200 and the coherent signals are with the same frequency. The simulation results for the comparison of incoming coherent and noncoherent signals employing MUSIC algorithm is shown below:

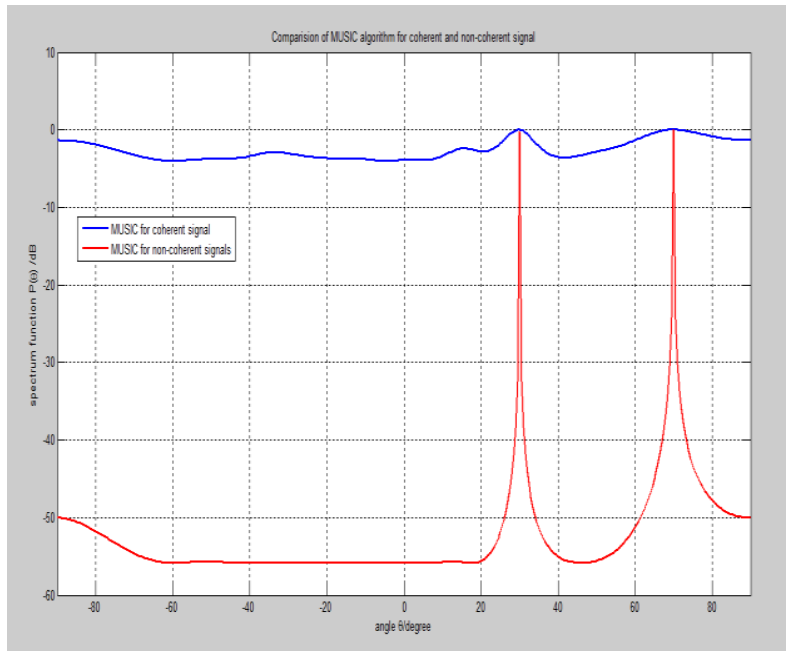

Fig.10. Estimation of coherent and non-coherent incoming signals using MUSIC algorithm.

The blue and red curves correspond to the estimation curves for coherent and non-coherent incoming signals. It can be clearly seen that the MUSIC algorithm cannot detect the related signals with the same frequency. The peak search method fails when the signals are coherent. Hence the estimation of the related signal i.e. the signals which are coherent cannot be efficiently carried out using the basic conventional MUSIC algorithm. This algorithm declines in its performance and accuracy when the incoming signals are related and even when there are amplitude as well as phase errors.

\section{THE IMPROVED MUSIC ALGORITHM}

The new Improved MUSIC algorithm which is proposed in the following section can effectively estimate the related as well as the non-related signals. It can even partially calibrate the array errors. As the peaks obtained are not sharp and narrow, they fail to estimate the arrival angle for coherent signals. So we need to move towards an improved MUSIC algorithm to meet the estimation requirements for coherent signals.

In order to improve the estimation results for the conventional MUSIC algorithm [15], we need to introduce an identity transition matrix ' $\mathrm{I}$ ' to the matrix ' $\mathrm{X}$ ' which corresponds to the received signal. On introducing the identity matrix we get:

$$
\mathrm{X}=\mathrm{IO}^{*}
$$

$\mathrm{O}^{*}$ in the above equation represents the complex conjugate of the received signal matrix ' $\mathrm{O}$ '. If ' $\mathrm{C}_{\mathrm{X}}$ ' denotes the correlation function for the received signal matrix, the modified ' $\mathrm{C}_{\mathrm{X}}$ ' on introducing the identity is given as:

$$
\mathrm{C}_{\mathrm{X}}=\mathrm{E}\left[\mathrm{X} \mathrm{X}{ }^{\mathrm{H}}\right]=\mathrm{TC}_{\mathrm{O}}{ }^{*}
$$

A reconstructed matrix ' $C$ ' is given by summing up $C_{X}$ and $\mathrm{C}_{\mathrm{O}}$.As the matrix are summed up they will have the same noise subspaces:

$$
\begin{gathered}
\mathrm{C}=\mathrm{C}_{\mathrm{O}}+\mathrm{C}_{\mathrm{X}} \\
\mathrm{C}=\mathrm{AC}_{\mathrm{S}} \mathrm{A}^{\mathrm{H}}+\mathrm{I}\left[\mathrm{AC}_{\mathrm{S}} \mathrm{A}^{\mathrm{H}}\right]^{*} \mathrm{I}+2 \sigma^{2} \mathrm{I}
\end{gathered}
$$

The new improved MUSIC is different from the conventional one as it filters out the noise subspace obtained after decomposition of $\mathrm{C}_{\mathrm{O}}$ and uses the new noise subspace for the spectral estimation which is obtained by the characteristic decomposition of the resultant matrix $\mathrm{C}$ is used for the spatial spectrum construction and to obtain peaks.

On keeping all the specifications to be the same as in the simulations for basic MUSIC algorithm, we carry out the same for improved MUSIC. The graph shown below gives a comparison between the estimation of coherent and non coherent signals using the new improved MUSIC algorithm.

In the following figure it can be seen that the Improved MUSIC eliminates the limitations of the MUSIC algorithm. The performance of the improved MUSIC for the estimation of coherent signals is satisfactory. The graph even shows that the resolution and accuracy of the Improved MUSIC for non-coherent signals is quite more than that for the coherent signals 


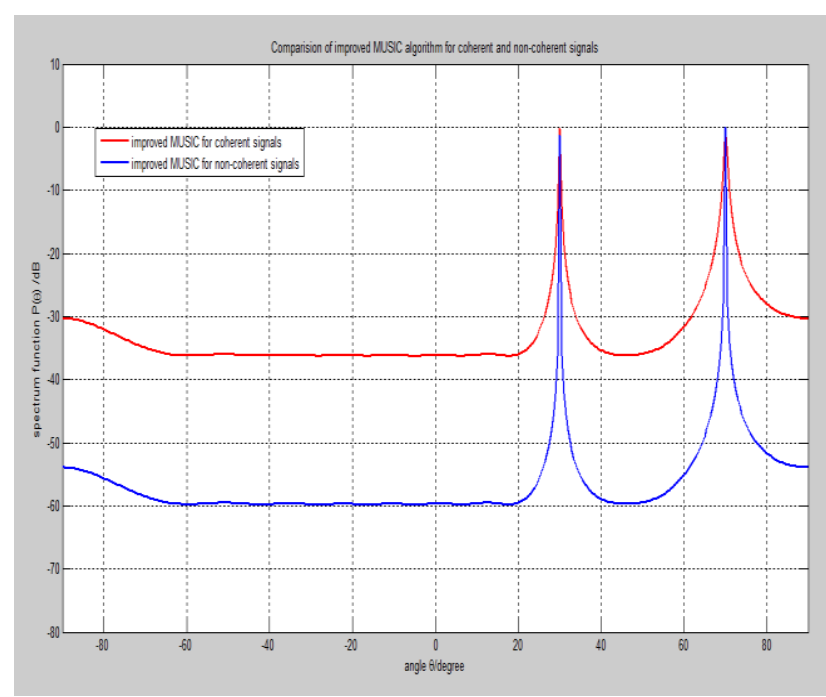

Fig.11. Estimation of coherent and non-coherent incoming signals using Improved MUSIC algorithm.

The red and blue curves give the estimation results for coherent and non-coherent signals respectively. The blue curves corresponding to the non-coherent signals cover a larger range of spectrum and hence the improved MUSIC is more efficient for non-coherent than those of the incoming coherent signals. The upcoming figure compares both MUSIC and Improved MUSIC algorithm for the estimation of coherent signals.

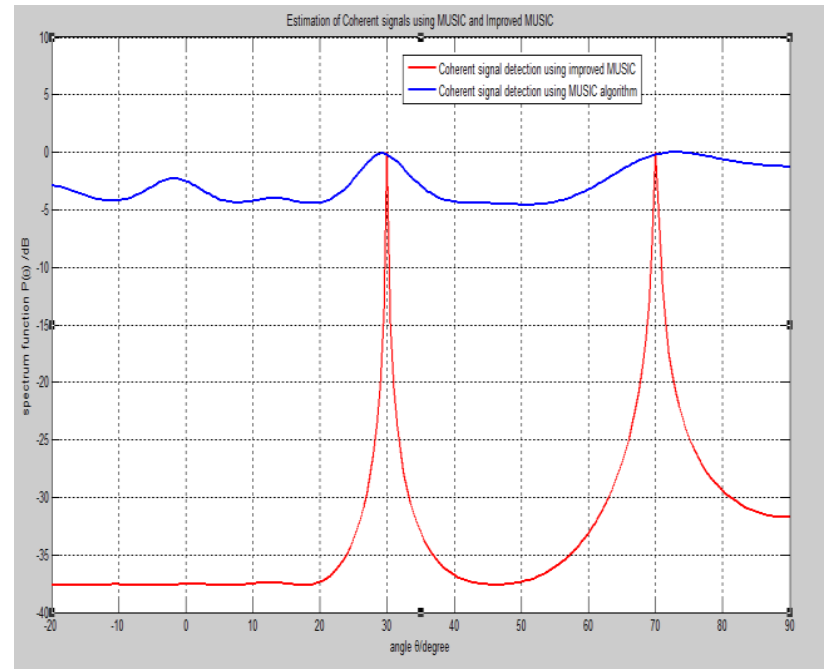

Fig.12. Comparison of MUSIC and Improved MUSIC for the estimation of coherent signals.

So the problems in estimating the coherent signals are overcome by the Improved MUSIC algorithm. The newly introduced Improved MUSIC even shows better performance for the estimation of non-coherent signals. This can be efficiently judged by comparing the simulation curve for the estimation of the arriving noncoherent signals using both MUSIC and Improved MUSIC.

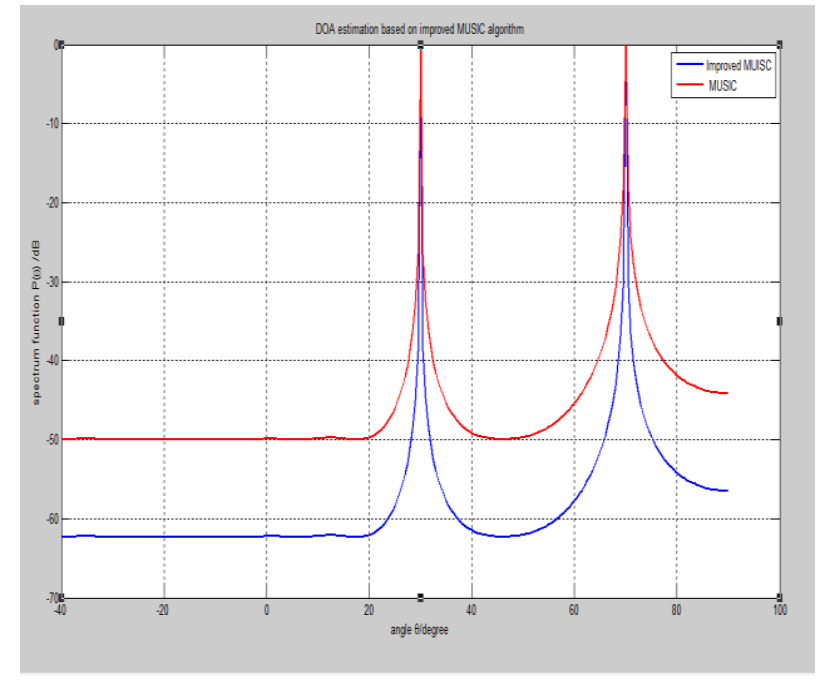

Fig.13. Estimation of incoming non-coherent signals using both MUSIC and Improved MUSIC algorithms.

The figure shows the same where the blue curve representing the Improved MUSIC estimation plot covers a wider spectral range than the blue curve corresponding to the conventional one. So the non-coherent signals can also be estimated with higher resolution and accuracy with the use of Improved MUSIC algorithm.

The efficiency of this improved MUSIC for both coherent and non-coherent signals can also be increased by varying the parameters as in the case of the basic MUSIC algorithm. Increasing the number of sensors, number of snapshots, increasing the inter element spacing and SNR can bring about better efficiency in Improved MUSIC as well.

\section{CONCLUSION}

The eigen values and eigen vectors forms the base for both MUSIC and improved MUSIC algorithms. They exploit the orthogonality property between the signals and the noise subspaces to estimate the direction of arrival of the incoming signal. Performance of these algorithms can be increased with an increase in the number of array elements, on considering a larger number of snapshots, increasing the element spacing with some above mentioned constrained and effectively increases on increasing the value of SNR.

The MUSIC algorithm is found to be efficient for the detection of non-coherent signals and the performance declines sharply when the incoming signals are coherent in nature. The Improved MUSIC algorithm caters the problem for the estimation of coherent signals.

\section{REFERENCES}

[1] Haykin S,Reilly JP, Vertascitsch E. Some Aspects of Array Signal Processing. IEE Proc. F,1992,139; p1 26. 
[2] Zekeriya Aliyazicioglu, H.K. Hwang. Marshall Grice, Anatoly Yakovlev, "Sensitivity analysis for direction of arrival estimation using a Root-MUSIC algorithm", Proceedings of the International Multi Conference of Engineers and Computer Scientists vol II IMECS, 19-21 March 2008

[3] T. S. Rappaport and J. C. Liberti Jr.," Smart Antennas for Wireless Communications: IS-95 and Third Generation CDMA Applications", Upper Saddle River, NJ: Prentice Hall, 1999

[4] Yongliang Wang, " Space Spectral Estimation Theory and Algorithm”, China. Tsinghua Press, 2004.

[5] Richard Roy, ThonasKailath, "ESPRIT-Estimation of Signal Parameters via Rotational Invariance Techniques",IEEE Trans on Acoustics Speech and Signal Processing, vol. 37. No.7 pp 984 995, July 1989.

[6] Ronald D, Degrot., "The Constrained MUSIC Problem" ,IEEE Trans on SP.1993. vol. 41(3). P1445 1449.

[7] Fuli Richard,"Analysis of Min-norm and MUSIC with Arbitrary Array Geometry", IEEE Trans on AES.1990. vol. 26(6). P976 985.

[8] M.Gavish, "Performance Analysis of the VIA ESPRIT Algorithm”, IEE-Proc-F. 1993. vol. 140(2). P123 128.

[9] B. P. Kumar, G. R. .Branner, "Design of Unequally Spaced Arrays for Performance Improvement", IEEE Trans. On Antennas Propagation, 47 (3): 511-523, 1999.

[10] Y.J Huang, Y.W Wang, F.J Meng, G.L Wang, “A Spatial Spectrum Estimation Algorithm based on Adaptive Beam forming Nulling” ,Piscataway, NJ USA. Jun, 2013. Pp 220-4.

[11] ZHNAG Hongmei, GAO Zhenguo, FU Huixuan, "High Resolution Random Linear Sonar Array Based MUSIC Method for Underwater DOA Estimation” Proceeding of 32nd Chinese Control Conference, July 26-28,2013.

[12] Ralph O, Schmidt, "Multiple Emitter Location and signal Parameter Estimation", IEEE Trans. On Antennas and Propagation, March 1986. vol. 34. No. 3. pp 276-280.
[13] L.N Yan, " Study of Factors Affecting Accuracy of DOA Modern Rader" ,June 2007. vol. 29. No. 6. pp 70-3.

[14] Fei Wen, Qun Wan, Rong Fan, Hewen Wei, "Improved MUSIC Algorithm for Multiple Noncoherent Subarrays" ,IEEE Signal Processing Letters, vol. 21, no. 5, May, 2014

[15] Debasis Kundu, "Modified MUSIC Algorithm for estimating DOA of signals", Department of Mathematics Indian Institute of Technology, Kanpur, India. November 1993.

\section{Authors' Profiles}

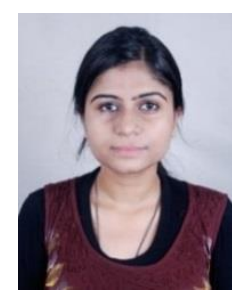

Pooja Gupta was born in Odisha, India in 1992. She has completed her Dual Degree B.Tech and M.Tech programme in Electronics and Telecommunication Engineering in 2015 from KIIT University, Bhubaneswar, Odisha. She is currently working as a Faculty Associate at KIIT University. Her research area includes subspace methods for Direction of Arrival Estimation.

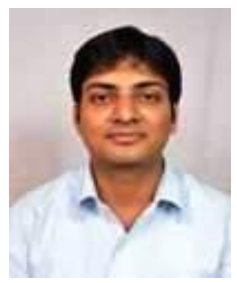

Vijay Kumar Verma was born in Uttar pradesh, India on 21 May, 1990 and has completed his post graduation from IIT(BHU) Varanasi. He is currently working as an Assistant Professor at KIIT University, Odisha. His field of interest includes magnetic levitation systems, optimization techniques.

How to cite this paper: Pooja Gupta, Vijay Verma,"Optimization of MUSIC and Improved MUSIC Algorithm to Estimate Direction of Arrival", International Journal of Image, Graphics and Signal Processing(IJIGSP), Vol.8, No.12, pp.30-37, 2016.DOI: 10.5815/ijigsp.2016.12.04 